\title{
Investigation of journal orbit and flow pattern in a dynamically loaded journal bearing
}

\author{
Christiansen, Christian Kim; Walther, Jens Honore; Klit, Peder; Vølund, Anders
}

Published in:

Tribology International

Link to article, DOI:

10.1016/j.triboint.2017.04.013

Publication date:

2017

Document Version

Peer reviewed version

Link back to DTU Orbit

Citation (APA):

Christiansen, C. K., Walther, J. H., Klit, P., \& Vølund, A. (2017). Investigation of journal orbit and flow pattern in a dynamically loaded journal bearing. Tribology International, 114, 450-457.

https://doi.org/10.1016/j.triboint.2017.04.013

\section{General rights}

Copyright and moral rights for the publications made accessible in the public portal are retained by the authors and/or other copyright owners and it is a condition of accessing publications that users recognise and abide by the legal requirements associated with these rights.

- Users may download and print one copy of any publication from the public portal for the purpose of private study or research.

- You may not further distribute the material or use it for any profit-making activity or commercial gain

- You may freely distribute the URL identifying the publication in the public portal 


\title{
Investigation of journal orbit and flow pattern in a dynamically loaded journal bearing
}

\author{
Christian Kim Christiansen ${ }^{\mathrm{a}, \mathrm{d}, *}$, Jens Honore Walther ${ }^{\mathrm{a}, \mathrm{b}}$, Peder Klit ${ }^{\mathrm{a}}$, \\ Anders Vølund ${ }^{\mathrm{c}}$ \\ ${ }^{a}$ Department of Mechanical Engineering, Technical University of Denmark, Nils Koppels \\ Allé, bld. 404, 2800 Kgs. Lyngby, Denmark \\ ${ }^{b}$ Computational Science $\mathcal{E}$ Engineering Laboratory, ETH, Clausiusstrasse 33, Zürich, \\ CH-8092, Switzerland \\ ${ }^{c}$ MAN Diesel 83 Turbo SE, Teglholmsgade 41, 2450 Copenhagen SV, Denmark \\ ${ }^{d}$ Center for Bachelor of Engineering Studies, Technical University of Denmark, \\ Lautrupvang 15, 2750 Ballerup, Denmark
}

\begin{abstract}
A hydrodynamic journal bearing has been investigated using both the traditional two-dimensional (2D) Reynolds equation, and the full solution being the three-dimensional (3D) Navier-Stokes equations.

The two approaches are compared by performing an investigation of two inlet groove designs: the axial and the circumferential groove, respectively, on a bearing with length-to-diameter ratio of 0.5 exposed to a sinusoidal load pattern. Pressure distributions, journal orbits and frictional losses are compared. The modelling of grooves by pressure boundary conditions versus geometric conditions is examined. It is investigated if the presence of a groove increases frictional losses and the increase relates to groove dimensions. Furthermore, the influence of the groove design on the flow field is studied using the 3D solution.
\end{abstract}

Keywords:

journal, simulation, Navier-Stokes equations, Reynolds equation

\footnotetext{
*Corresponding author

Email address: chkch@dtu.dk (Christian Kim Christiansen)
} 


\section{Introduction}

Ever since Reynolds described the governing equation of hydrodynamic lubrication in 1886 [1], computing the solution to what has become known as the Reynolds equation, has occupied the scientific branch with roots in the fields of engineering and mathematics. Being a partial differential equation, most analytical solutions cannot be established. For his paper, Reynolds applied assumptions on an infinitely long bearing and a series expansion to perform the integration. This assumption only holds for low eccentricity ratios $(<0.5)$. With the advent of a particular substitution in 1904 Sommerfeld was able to obtain a closed form solution to the case Reynolds had tested [2]. In 1952 Ocvirk used the opposite approach by assuming the bearing to be infinitely narrow [3]. These two approaches have one major drawback, despite softening the requirements to what is considered infinitely long and short, that is most bearings fall between those definitions. For such bearings only a numerical solution is possible.

With the appearance of computers occurring after World War II, the numerical methods became more and more feasible, especially for solving 1- and 2D problems like Reynolds equation. Simultaneously, with the continuously improving computational power being available, focus turned onto improved modelling of the film rupture boundaries independently started by Swift and Stieber in the 1930's [4, 5]. As the extent of the film has proven important in terms of the dynamic properties of the bearing, a significant attention has been put into the development of theoretical models and their numerical implementation $[6,7]$.

During the last decades the ever-increasing trend in computing power have made 3D analysis possible. Obviously, analysis would include the pressure distribution, and further, more advanced problems like thermal distribution in lubricant [8] and modeling of Bingham lubricants [9] have been investigated. Recently, Schmidt et al. have have used the open source CFD code OpenFOAM for investigations on journal bearings [10, 11, 12].

In the present paper, a comparison between a simplified (2D), but fast, finite element code solving Reynolds equation and a slower, but physically more correct Navier-Stokes based finite volume code is performed. The latter is important when investigating more complex designs for bearing feed grooves violating the typical assumptions leading to Reynolds equation. To the author's knowledge, the dynamic loading of a journal bearing is a special case that has not received attention in terms of solution by a commercial 
CFD code based on the Navier-Stokes equations. Using a Reynolds equation based code Cheng and Ji also impose a dynamic load, but do not have the possibility of modelling deep grooves violating Reynolds equation [13]. Other works employ sophisticated cavitation algorithms in commercial codes like ANSYS CFX with Song and Gu solving a thermal problem [14], or Fluent with Zhang et al. determining stiffness coefficients [15], and purpose built codes with Besanjideh and Nassab analysing compressibility [16], but all only for static load cases. Thus, this paper aims at applying a commercial Navier-Stokes code to solve the dynamically loaded bearing with a simple cavitation model (half-Sommerfeld).

\section{Methodology}

Being a problem of fluid dynamics, the Navier-Stokes and continuity equations are the governing relations for the case of hydrodynamic lubrication:

$$
\begin{gathered}
\rho\left(\frac{\partial \boldsymbol{u}}{\partial t}+\boldsymbol{u} \cdot \nabla \boldsymbol{u}\right)=-\nabla p+\nabla \boldsymbol{T} \\
\frac{\partial \rho}{\partial t}+\nabla \cdot(\rho \boldsymbol{u})=0
\end{gathered}
$$

Here $\rho$ is density, $\boldsymbol{u}$ is the velocity vector, $\nabla$ is the vector differential operator $\nabla=\{\partial / \partial x, \partial / \partial y, \partial / \partial z\}^{T}, p$ is pressure, $\boldsymbol{T}$ is the deviatoric stress tensor $\left(\boldsymbol{T}=\mu\left(\nabla \boldsymbol{u}+(\nabla \boldsymbol{u})^{T}-2 / 3(\nabla \cdot \boldsymbol{u})\right)\right.$ with $\mu$ being the dynamic viscosity). Assuming isothermal conditions, the equation of state applied is:

$$
\rho(p)=\rho_{0}+\frac{p_{r e l}}{v_{s}^{2}}
$$

The relative pressure is defined as the difference between absolute and atmospheric pressure, $p_{r e l}=p-p_{a t m}$, and $v_{s}$ is the sonic speed of the lubricant. The term $p_{\text {rel }} / v_{s}^{2}$ originates from integration of the definition of the speed of sound, $v_{s}^{2}=\partial p / \partial \rho$. The fraction is hence $p_{\text {rel }} / v_{s}^{2}$ is hence a compressibility term added to the incompressible density $\rho_{0}$. Equation (3) is suggested by the developers of the finite volume code, CD-adapco [17].

The traditional approach for solving the hydrodynamic lubrication problem is a simplification to the Navier-Stokes equations. Applying the assumptions of a thin lubricant film and isothermal conditions to the Navier-Stokes 


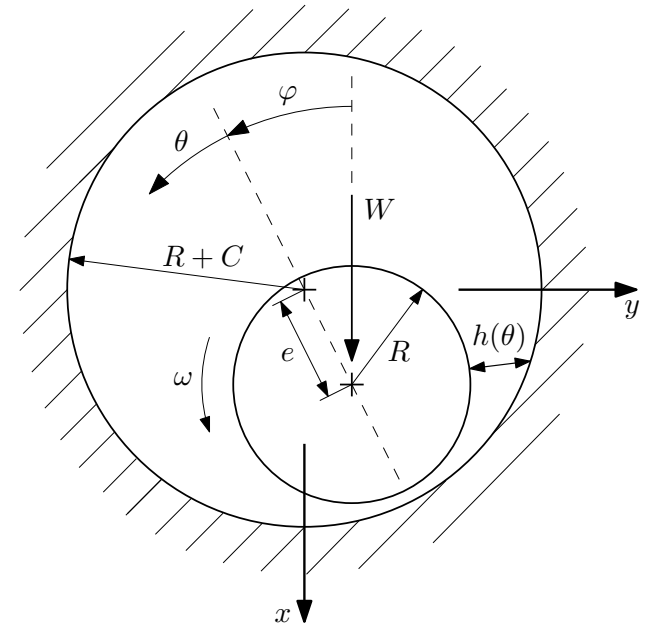

(a)

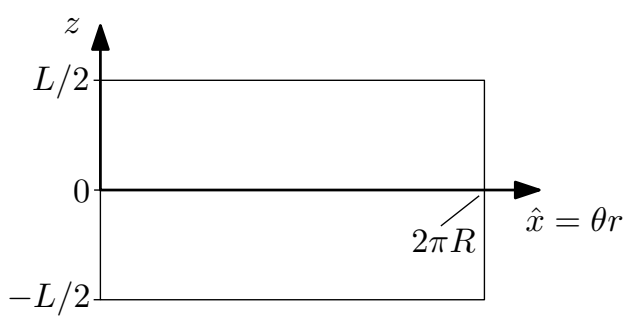

(b)

Figure 1: Simple inlet groove. (a) Bearing geometry and imposed boundary conditions. Axial coordinates $z$ and $\zeta$, with $\zeta$ from Equation (4), is pointing out of the plane. (b) Unfolded bearing geometry.

equations, Reynolds equation is found [1]. Below, it is shown in its nondimensional form as applied to the numerical implementation:

$$
\frac{\partial}{\partial \theta}\left(\bar{h}^{3} \frac{\partial \bar{p}}{\partial \theta}\right)+\frac{\partial}{\partial \zeta}\left(\bar{h}^{3} \frac{\partial \bar{p}}{\partial \zeta}\right)=\frac{\partial \bar{h}}{\partial \theta}+\frac{2 \omega^{*}}{\omega} \frac{\mathrm{d}}{\mathrm{d} \bar{t}} \bar{h}
$$

Here $\theta=\hat{x} / R$ and $\zeta=z / R$ are circumferential and axial directions, respectively, $\bar{h}=h / C$ is the non-dimensional film thickness, $\bar{p}=p /\left(6 \mu \omega(R / C)^{2}\right)$ is the non-dimensional pressure, $\omega$ is angular (relative) velocity, $\omega^{*}$ is a characteristic angular velocity (in this work chosen as the angular velocity of the journal) and $\bar{t}=t \omega^{*}$ is non-dimensional time. A typical application of Reynolds equation is the journal bearing seen in Figure 1, where also the linear length and width coordinates $\hat{x}$ and $z$ together with journal radius $R$ and radial bearing clearance $C$ are shown, and $t$ designates time.

Both approaches; the Navier-Stokes (NS) equations, Equations (1)-(2), and the Reynolds equation (RE), Equation (4), predict an increase in pressure, followed by a decrease to the same pressure magnitude, such that the amplitude is anti-symmetric, due to the converging-diverging geometry. Besides for submerged and/or very lightly loaded bearings, this anti-symmetry is lost due to dissolution of gasses and cavitation effects. The cavitation modelling in this paper, is basically what is known as the half-Sommerfeld 
solution [18], but instead of neglecting all negative pressures, only pressures below the saturation pressure $p_{\text {sat }}$ is modified to obtain an effective pressure, $p_{\text {eff }}$, as explained in Equation (5). It is a very simplified consideration to cavitation and does not preserve the continuity (mass flow) through the cavitated zone [18]. The reason for using the half-Sommerfeld solution is the easy implementation, particularly in the Navier-Stokes code with a pre-compiled solver suite. Before resorting to the half-Sommerfeld solution both RayleighPlesset and degassing models have been tried out, but as Braun and Hannon conclude in their review, those models are not yet readily available [19].

$$
p_{\text {eff }}= \begin{cases}p & \text { if } p>p_{\text {sat }} \\ p_{\text {sat }} & \text { if } p \leq p_{\text {sat }} .\end{cases}
$$

To solve the dynamic lubrication problem, the two approaches deviate as described in the following sections.

\subsection{Discretisation}

\subsubsection{Finite volume}

The governing Navier-Stokes equations (Equations (1)-(2)) are discretised by a finite volume scheme and then solved with the commercial code STAR$\mathrm{CCM}+$ version 10.02. A full 3D solution is obtained. The dynamic squeeze term $\mathrm{d} h / \mathrm{d} t$ is modelled with a build-in 6-DOF solver applying Newton's second law to relate surface forces (lift) and external forces (imposed loads) with a resulting translational and rotational motion [17].

\subsubsection{Finite element}

Equation (4) is solved with a purpose written, MATLAB based finite element code using triangular first order elements. This solution is only 2D (no variation in thickness direction). The dynamic squeeze term $\mathrm{d} h / \mathrm{d} t$ is modelled with Lund's perturbation method [20] and the time integration is a simple Euler method. Further details on the implementation and a verification are given in [21].

\section{Numerical setup}

\subsection{The journal bearing}

The bearing chosen for the simulations is described by Table 1 and illustrated in Figures 2 and 3. Figure 3a shows the axial groove and Figure 3b 


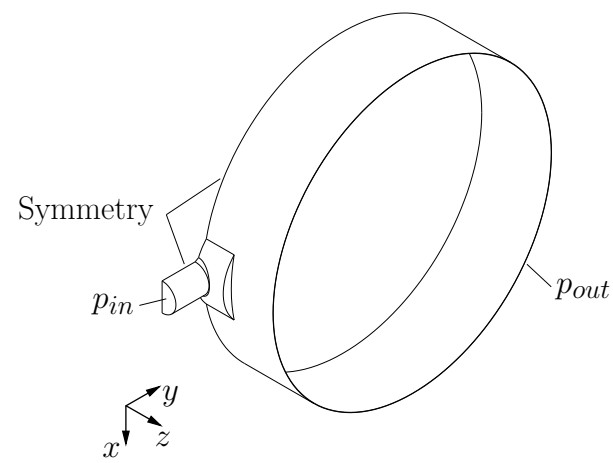

Figure 2: Bearing geometry and imposed boundary conditions. Refer to Table 1 for values..

shows the circumferential groove. In either case, symmetry is exploited in the axial direction and it is assumed that the bearing housing is infinitely rigid. Lubricant is fed in at the inlet and exiting the bearing at the free edge. The pressure conditions are listed in Table 1 as well as the angular velocity of the journal. All walls are modelled with no-slip condition.

As imposed load $\left(W_{x}(t)\right)$ on the bearing, a cosine function is chosen,

$$
W_{x}(t)=\frac{1}{2}\left[1-\cos \left(2 \pi \cdot \frac{t}{T_{p}}\right)\right] \cdot A
$$

where $T_{p}=0.2 \mathrm{~s}$ is the duration of one revolution and $A=3 \times 10^{3} \mathrm{~N}$ is the amplitude. To simplify the computations, a shift to the cosine-based load function is applied such that $W_{x}(0)=0$ and $W_{x}(t) \geq 0$. These choices have two advantages; $i$ ) it eliminates the need for ramping up the load as this is built into the function itself and $i$ ) the positive load only forces the journal downwards, simplifying the stability requirements to the computations as the orbit will be restricted compared to a sign-alternating function. Item ii) also simplifies the loading arrangement in case of an experimental setup, as only tensional or compressional loading will be required.

The excitation frequency is chosen to be half the rotational frequency to have a time-varying force not being too strong with regards to computational stability, but still allow for some revolutions of the bearing within a manageable time frame. This concern on the stability relates to the Navier-Stokes code, where a low variation in load versus time step was experienced as a requirement to avoid large displacement increments yielding collapsed cells. Total simulated time $\left(t_{\text {end }}\right)$ is chosen, by setting $t_{\text {end }}=0.4 \mathrm{~s}$, such that two 


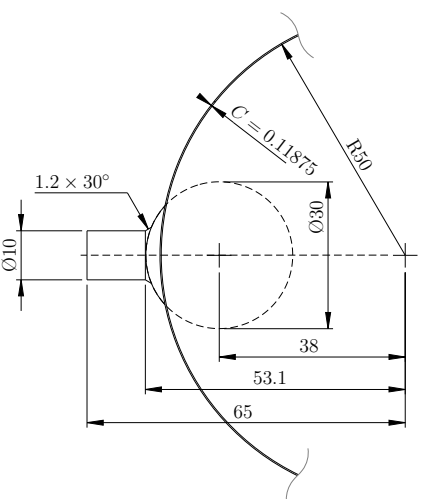

(a)

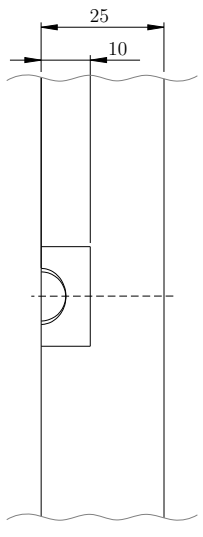

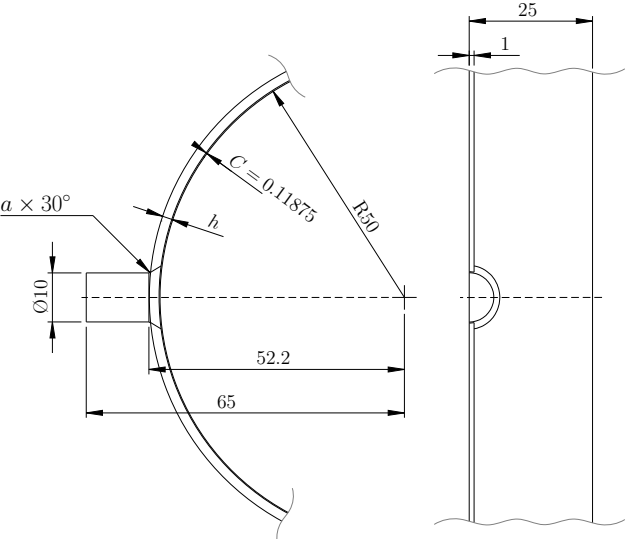

(b)

Figure 3: Specification of grooves - dimensions in mm. (a) Axial groove. (b) Circumferential groove. The groove height is varied as $h=\{2.0 ; 0.5 ; 0.1\} \mathrm{mm}$ in the circumferential case, hence the variable $a$ for the chamfer (recall, bearing clearance is $C=0.11875 \mathrm{~mm}$ ).

periods of the load function is imposed on the bearing during four revolutions. In this way a periodic solution for the journal orbit should be obtained, even from an arbitrary starting position.

The lubricant is considered to be Newtonian and with the properties given in Table 2.

\subsection{Finite volume}

The two main advantages for the application of a commercial code like STAR-CCM+ is the ability to choose between several approaches to a numerical representation of the physical problem. Particularly, this relates to the cavitation modelling where two methods have been investigated as mentioned in Section 2. Also the mesh generation in the thin film is non-trivial.

For the simulations both first and second order upwind schemes have been tested for the discretisation of the convection term. Likewise, the grid fluxes have been integrated wrt. time using both first and second order methods. From a stability point of view, the first order methods have been preferred, as the small time steps encountered eliminate accuracy issues, particularly in the time integration. 
Table 1: Dimensional and operational parameters for the journal bearing.

\begin{tabular}{lccc}
\hline Parameter & Symbol & Value & Unit \\
\hline Length & $L$ & 50 & $\mathrm{~mm}$ \\
Diameter & $D$ & 100 & $\mathrm{~mm}$ \\
Clearance (radial) & $C$ & 118.75 & $\mu \mathrm{m}$ \\
Mass of journal & $m$ & 1.53 & $\mathrm{~kg}$ \\
Rotational speed & $\omega$ & 600 & $\mathrm{RPM}$ \\
Load (vertical) & $W_{x}$ & $W_{x}(t)^{\mathrm{a}}$ & $\mathrm{N}$ \\
Inlet pressure & $p_{\text {in }}$ & $2 \times 10^{5}$ & $\mathrm{~Pa}$ \\
Outlet pressure & $p_{\text {out }}$ & $1 \times 10^{5}$ & $\mathrm{~Pa}$ \\
\hline
\end{tabular}

a See Equation (6).

Table 2: Lubricant properties.

\begin{tabular}{lccc}
\hline Parameter & Symbol & Value & Unit \\
\hline Dynamic viscosity & $\mu$ & 0.071 & $\mathrm{Pas}$ \\
Saturation pressure & $p_{\text {sat }}$ & $90 \times 10^{3}$ & $\mathrm{~Pa}$ \\
Density & $\rho_{0}$ & 885 & $\mathrm{~kg} / \mathrm{m}^{3}$ \\
Speed of sound & $v_{s}$ & 1300 & $\mathrm{~m} / \mathrm{s}$ \\
\hline
\end{tabular}

\subsubsection{Time stepping}

Due to the constantly varying mesh, a varying time step is desirable to ensure an efficient simulation. With numerical stability in mind the desired Courant number $C r=U \Delta t / \Delta x$ is specified as $C r=1$. Further stability is sought introduced by defining an interval for the admissible time step $\Delta t \in\left[1 \times 10^{-6} \mathrm{~s}, 5 \times 10^{-5} \mathrm{~s}\right]$ and averaging between the calculated (new) step and the current one, $t_{\text {new }}=\alpha t_{\text {old }}+(1-\alpha) t_{\text {new }}$. The time range is chosen as to limit the Courant number to $C r_{\max } \approx 1$ for the expected grid sizes and velocities during the simulation, and the averaging weight $\alpha=0.5$.

\subsubsection{Motion}

As explained in Section 2, the motion is computed in a dynamic fluid body interaction (DFBI) fashion. With six degrees of freedom available (translation and rotation in all three directions), a set of limitations have to be applied. Therefore, to mimic the bearing, only in-plane translation $(x, y)$ is allowed, cf. Figure 2. The rotation around $z$ is specified as a velocity bound- 
ary condition on the journal wall. The journal mass, $m$, listed in Table 1, is based on the half-length journal due to the symmetry boundary condition applied. During post-processing, the assumption of $m \cdot \ddot{x}<<W$ is proved valid as $m \cdot \ddot{x}_{\max } \approx 0.01 \cdot W_{\max }$.

\subsubsection{Mesh}

Despite a relatively simple geometry, the mesh generation is non-trivial. Constituted of a thin and a bulky part, in which, from theoretical expectations, various flow phenomena might occur, the bearing presents some difficulties. Due to the non-linear problem, and hence iterative solution method, together with integration in time making the problem very computational demanding, much effort has been put into the optimisation of the mesh. For better control of the meshing process, the bearing with the axial groove as seen in Figure 3a, is split up into two groups; one for the film and one for the inlet and groove (the circumferential groove model in Figure 3b is split into three; inlet pipe, groove and film). This makes it possible to obtain a suitable mesh taking advantage of the geometry.

Film. With the geometry being pre-dominantly thin, advantage of STARCCM+'s build-in thin mesher can be taken. It is an unstructured mesher that allows for a larger surface/thickness aspect ratio to minimise the cell count in circumferential and axial directions, and just as important, is the radial mesh being structured (layered). A total of 10 cells is used to resolve the radial direction, cf. Figure $4 \mathrm{~b}$. The total cell count is approximately 596,000 cells. Based on the convergence of the orbit prediction, the medium case of 10 radial layers is used for the circumferential groove with varying groove height in the finite volume discretisation.

To reflect the calculated motions, the mesh is deforming while maintaining its topology (also known as 'morphing'). Specific cell quality parameters are monitored and work as stopping criteria to the simulation, if the mesh becomes too distorted. The applied mesh have proved quite robust and able to maintain an acceptable quality even with eccentricity ratios of $\varepsilon \equiv e / C \approx 0.7-0.8$.

Groove. The inlet groove and pipe is meshed with an unstructured polyhedral due to the geometry, see Figure 4a. Whereas the inlet pipe is expected to be dominated by a pipeflow, the groove is meshed to resolve a more complicated

flow. Two prism layers are used to improve the accuracy near walls. The total cell count is approximately 55,000 cells. 


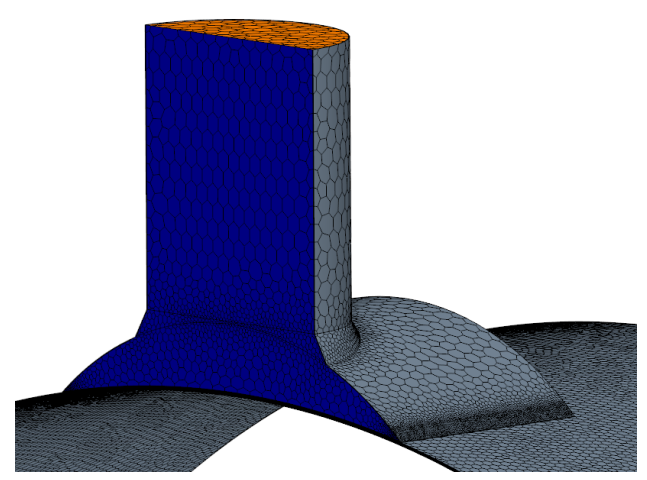

(a)

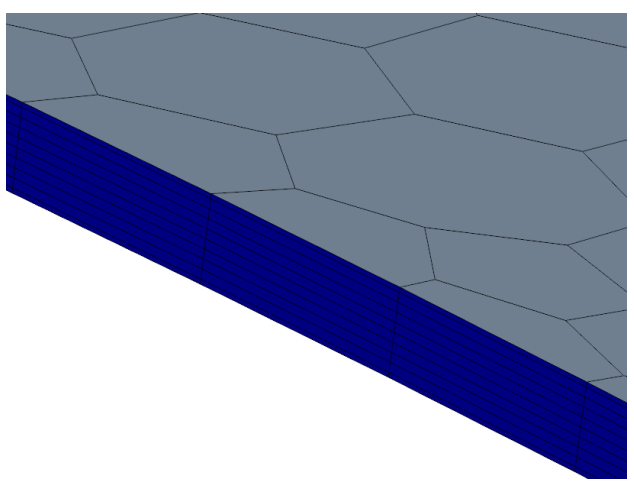

(b)

Figure 4: Finite volume mesh. (a) Overview of inlet pipe and film regions. (b) Structured, thin mesh in radial direction for the film region.

\subsubsection{Compressibility}

To avoid numerical instabilities in STAR-CCM+ in connection with the 6DOF solver, the lubricant is considered compressible. It appears that strong fluctuations in the dependent terms pressure field and journal translation velocity is smoothed out by allowing a weak compressibility of the lubricant. From the general equation of state, the density is a function of (local) relative pressure as given in Equation (3). Studying the fraction $p_{\text {rel }} / v_{s}^{2}$ in Equation (3) it is clear that for pressure values typical for journal bearings ( $50 \mathrm{MPa}$ is high in this context), the fraction is $p / v_{s}^{2} \approx 3 \mathrm{~kg} / \mathrm{m}^{3}$, such that the lubricant is only weakly compressible $\left(\rho_{0}=885 \mathrm{~kg} / \mathrm{m}^{3}\right)$.

\subsubsection{Cavitation}

The modified half-Sommerfeld treatment of Equation (5) is applied. Please, note this is not a standard model in STAR-CCM+, but the pressure solver allows a user specified value for the allowed minimum pressure. If the computed pressure in any cell drops below this threshold during the simulation, then the defined minimum values is assigned. The minimum value is set to $p_{\text {sat }}=90 \times 10^{3} \mathrm{~Pa}$, see also Table 2 .

\subsection{Finite element}

Contrary to the finite volume scheme solving the non-linear Navier-Stokes equations (Equation (1)), the finite element code is a much simpler set-up due to linearisation of the Reynolds equation (Equation (4)). The imposed 


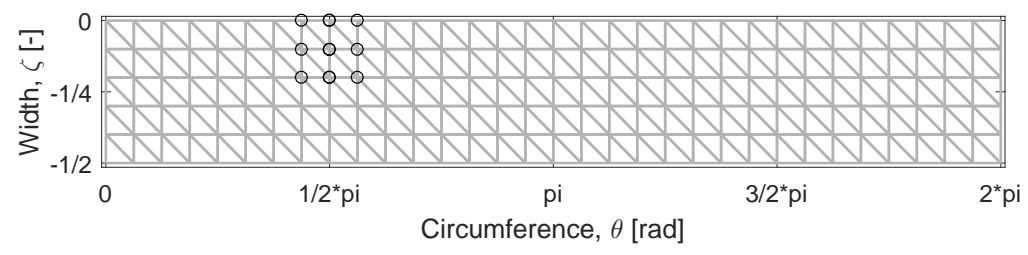

Figure 5: Illustration of finite element mesh. The groove is modelled by imposing inlet pressure on the nodes covering the groove, here represented by circles.

load of Equation (6) is discretised for every $1^{\circ}$ of journal rotation (compared to the $720^{\circ}$ of journal rotation during the simulation) to ensure a stable scheme due to the simple time integration. Thus, the time step is constant at $\Delta t=2.78 \times 10^{-4} \mathrm{~s}$.

The effect from the inlet is modelled onto the $2 \mathrm{D}$ domain by imposing inlet pressure on the affected nodes $\left( \pm 11.66^{\circ}\right.$ from horizontal center plane $)$ cf. Figure 5. This is done since the groove is much thicker than the surrounding film and hence not influenced by the pressure build-up suggested by Reynolds equation.

\subsubsection{Motion}

The motion of the journal is found from perturbations of a damper model of the journal. The damping coefficients relates to the forces and velocities in the manner:

$$
\left\{\begin{array}{l}
W_{x}-F_{x}\left(x_{i}, y_{i}, \dot{x}_{n}, \dot{y}_{n}\right) \\
W_{y}-F_{y}\left(x_{i}, y_{i}, \dot{x}_{n}, \dot{y}_{n}\right)
\end{array}\right\}=\left[\begin{array}{cc}
D_{x x} & D_{x y} \\
D_{y x} & D_{y y}
\end{array}\right]\left\{\begin{array}{c}
\Delta \dot{x}_{n} \\
\Delta \dot{y}_{n}
\end{array}\right\},
$$

where $W_{x}, W_{y}$ are the imposed load vector components, $F_{x}, F_{y}$ are the hydrodynamic load vector components, $D_{i j}, i=\{x, y\}, j=\{x, y\}$ are the damping coefficients and $\Delta \dot{x}_{n}, \Delta \dot{y}_{n}$ are the incremental velocity components of the journal. As $\boldsymbol{F}=\left\{F_{x}, F_{y}\right\}^{T}$ is a function of the velocities $\dot{x}, \dot{y}$, this is done iteratively; $\dot{x}_{n+1}=\dot{x}_{n}+\Delta \dot{x}_{n}$. For the next load step, a first-order Euler method is applied: $x_{i+1}=x_{i}+\Delta t \cdot \dot{x}_{i}$. Please, refer to [21, 20] for further information. As $\boldsymbol{F}=\boldsymbol{F}\left(x_{i}, y_{i}, \dot{x}_{n}, \dot{y}_{n}\right)$ one may argue, that the position $x$ could be taken as the unknown instead of $\dot{x}$, using the stiffness coefficients and position increment in the manner $F=D_{x x} \cdot \Delta x$. This is not done, in order to simplify computations by avoiding complications in obtaining two initial conditions for establishing journal velocity $\dot{x}_{i} \approx\left(x_{i}-x_{i-1}\right) / \Delta t$, as the velocity is otherwise unknown. 


\subsubsection{Mesh}

The mesh used in the finite element code is $\theta \times \zeta$ of $(30 \times 8),(60 \times 16)$, and $(90 \times 24)$, respectively, triangular and uniform elements.

Contrary to the finite volume discretisation, the modelling of groove extent is done by assuming a uniform, practically hydrostatic, pressure boundary condition imposed on the affected nodes in the finite element discretisation, and hence the three values of groove height are not 'seen' by the finite element discretisation leading to discrepancies.

\subsubsection{Cavitation}

The simple cavitation model described above is also implemented in the finite element code.

\section{Results}

In all the portrayed cases, the journal is rotating with $600 \mathrm{RPM}$ at the initial position $\varepsilon_{x}=\varepsilon_{y}=0.1$. The abbreviations RE and NS are used for the Reynolds equation and Navier-Stokes equations, respectively, while AG and CG designate the axial and circumferential grooves, respectively.

For the axial groove, a mesh refinement study is carried out, together with general visualisation of the flow field in and around the groove. The mesh refinement is performed by using the base element size of $1.5 \mathrm{~mm}$ and varying the number of thin mesher layers as 6,10 and 14, respectively, for the film region. The influence from a circumferential groove is investigated by varying the groove height as $h=2.0 \mathrm{~mm}, 0.5 \mathrm{~mm}$ and $0.1 \mathrm{~mm}$, respectively, corresponding to roughly 1,5 and 20 times the bearing clearance and using the medium mesh from the axial groove.

As only a single rotational speed of the journal is used, the Sommerfeld number $S$ given in Equation (8) is computed from Tables 1 and 2 to $S=0.21$. Being a non-dimensional bearing load, $S$ indicates that an increase in rotational speed will increase the load carrying capacity of the bearing (keeping other parameters fixed).

$$
S=\frac{\mu N D L}{W}\left(\frac{R}{C}\right)^{2}
$$

\subsection{Pressure distributions}

The pressure distribution of the four groove designs solved with NavierStokes are shown in Figure 6 after $3 / 2 \cdot T_{p}(t=0.3 \mathrm{~s})$. The differences in 
groove design is reflected in the pressure distributions. Considering the axial groove (Figure 6a), as reference, it is clear to see that with decreasing groove height, the three results converges towards the axial groove. This holds both for the 'tongue' seen around the inlet and the magnitude of maximum pressure. Figures $6 \mathrm{~b}$ and $6 \mathrm{c}$ also demonstrates the very low pressure of the groove, in terms leading to a confined region of very high pressure to obtain the same overall load carrying capacity. Figure 7 reproduces the influence of the circumferential groove reducing the bearing area. Comparing Figures $7 \mathrm{a}$ and $7 \mathrm{~b}$ to Figures $6 \mathrm{a}$ and $6 \mathrm{~b}$, respectively, shows good agreement, both in terms of maximum pressure and the overall pressure contours.

\subsection{Journal orbits}

The journal orbits for the two groove layouts are shown in Figure 8. Overall, there is a good agreement both in terms of magnitude and orbit shape for the two layouts. In terms of convergence studies, Figure 8a show fairly good agreement between the implementation of the Navier-Stoke and Reynolds equations regarding the axial groove. Contrary, especially the deep and shallow circumferential grooves of the Navier-Stokes solution is not well represented by the crude pressure approximation applied in the groove modelling in the discretisation of Reynolds equation, cf. Figure 8b. The consequences of the simplified approach taken to model the groove is clearly seen in Figure 8b. The general shape of the orbit is the same, but the position and magnitude of the orbit has moved relative to the initial position $\varepsilon_{x}=\varepsilon_{y}=0.1$. As those results are very different from the axial groove findings, an axial refinement $(\theta \times \zeta$ of $(60 \times 48))$ has been introduced to the finite element model to make sure only nodes being coincident with the groove position are included in the pressure boundary. However, it is clear from Figure 8a that the effect of the refinement is very limited.

With the same overall settings to the mesh, the variable groove height orbit is predicted in Figure 8b. Only for the shallow groove with groove height of $0.1 \mathrm{~mm}$, i.e. roughly the magnitude of the radial clearance, the orbit is only very similar to the one from the mesh-wise corresponding axial groove with 10 thin mesher layers in Figure 8a. Both this behaviour as well as the general pressure distributions of Figure 6 indicates the well known perception of grooves to violate the basic assumptions of Reynolds equation, to be true. In other words, only the shallowest of grooves possess some load carrying capacity. For both cases, the initial discrepancy described previously, results in an upwards movement of the journal, until load equilibrium is met. 
Table 3: Frictional losses.

\begin{tabular}{lccc}
\hline Groove type & $\mathrm{NS}$ & $\mathrm{RE}$ & Unit \\
\hline Axial groove $(90 \times 24)$ & -1.779 & -1.862 & $\mathrm{~N} \mathrm{~m}$ \\
Circ. groove $(20 \times C)$ & -2.273 & & $\mathrm{~N} \mathrm{~m}$ \\
Circ. groove $(5 \times C)$ & -2.110 & -2.273 & $\mathrm{~N} \mathrm{~m}$ \\
Circ. groove $(1 \times C)$ & -1.804 & & $\mathrm{~N} \mathrm{~m}$ \\
\hline
\end{tabular}

\subsection{Friction losses}

To quantify the suitability of the proposed bearing designs, friction loss is an obvious parameter. For the overall friction loss per revolution, the average frictional moment is used. In this work it is integrated numerically using the trapezoidal rule. With the somehow similar appearance of the various pressure distributions and journal orbits, only small deviations in the frictional moments are expected. Equation (9) relies on the film thichness $\bar{h}$ and pressure gradient $\partial \bar{p} / \partial \theta$ being summed up over the axial and circumferential bearing dimensions. Due to the minor differences, only a characteristic set of data are plotted in Figure 9.

$$
\bar{M}_{f}(t)=\frac{\pi}{L / D} \int_{0}^{L / D} \int_{0}^{2 \pi}\left(\frac{1}{\bar{h}(t)}+3 \bar{h}(t) \frac{\partial \bar{p}(t)}{\partial \theta}\right) \mathrm{d} \theta \mathrm{d} \zeta
$$

The trend of the four chosen cases are similar in pairs, i.e. the corresponding solution of Navier-Stokes and Reynolds equation. However, it is clear the deviation seen is caused in the first half of the intervals with increasing load and reaching its maximum very close to the midpoint of the interval. With the load curve being a sinusoidal function, this relates to areas with accelerating load increase (positive time derivative). Similarly, the second half of the interval with a decelerating load increment minimises the discrepancy and brings it to a low level for the entire interval of decreasing load. During the next load increase, the pattern repeats. Please recall the initial position of the journal $\left(\varepsilon_{x}=\varepsilon_{y}=0.1\right)$ does not coincide with the repeatable part of the orbit. This discrepancy decays after approximately $0.05 \mathrm{~s}$ according to Figure 9, after which the behaviour is periodic. The frictional losses over the two load cycles are listed in Table 3.

Though similarities between the Reynolds and Navier-Stokes solutions are to be expected from the orbits and pressure distributions, some conclusions can be drawn. Assuming the full 3D solution represented by the 
finite volume scheme to be the most correct approximation, the friction loss is overestimated by $5 \%$ for the axial groove and $26 \%$ for the circumferential groove by the finite element discretisation. With reference to the above results, the fine grid has resolved the problem sufficiently, such that the difference is more related to the choice and implementation of 2D (Reynolds equation) versus 3D (Navier-Stokes equations). Within the circumferential groove with the varying groove height, there is quite a difference $(26 \%)$ of the three heights. Overall, the larger groove (width and/or height) will result in increased frictional losses due to reduction of the effective bearing area.

\subsection{Flow fields}

Figure 10 illustrates the velocity fields of the two cases (axial groove and circumferential groove) in the symmetry plane. The importance of the groove design is clear from a comparison of the axial groove with the three different heights of the circumferential groove in Figure 10. Analysing the axial groove in Figure 10a it is clearly seen that one major vortex is formed at the top of the groove, just after the inlet pipe. Its clockwise rotation helps the inlet flow mixing with that of the rotational flow attached to the journal. This is also seen from the thin film section where the journal surface velocity driving the system is extending into the groove. Especially the deeper circumferential grooves of Figures 10b and 10c reveal how larger flow velocities tangential to the journal exist in the groove. For the specific case of Figure 10b, the counter clockwise rotating vortex will lead to a flow in clockwise direction in the groove, something that will extend the mixing area of the inlet from the inlet itself to a part of the groove as well. Primarily, the grooves are containing regions of very slow moving lubricant, corresponding to the grooves being deep enough to isolate the lubricant in the groove from the pressure generating film region. The smallest considered groove height of $0.1 \mathrm{~mm}$ results in a flow virtually identical to the axial groove, cf. Figures 10a and $10 \mathrm{~d}$, supporting the findings of the previous sections.

\subsection{Computational time}

Comparing the computational time for the simulation of the circumferential groove, Reynolds equation is solved in approx. 1 hour on a single node (only pressure), whereas the Navier-Stokes solver uses 72 days on 32 nodes in a cluster (pressure +3 velocity components). This big difference of walltime is reduced when converted to CPU time per degree of freedom, yielding $t_{C P U} \approx 3 \mathrm{~s} / \mathrm{DOF}(\mathrm{RE})$ and $t_{C P U} \approx 60 \mathrm{~s} / \mathrm{DOF}$ (NS), respectively. 
The main reason for this, is not the time required for solving the equations (in fact they are believed to be roughly equal), but the Navier-Stokes employs a much finer mesh. The finer mesh puts an upper bound on the time step to satisfy the desired Courant number $C_{r}=1$. Recall that $\Delta t_{\max }=5 \times 10^{-5} \mathrm{~s}$ in Subsection 3.2.1, whereas Reynolds equation utilises $\Delta t=2.78 \times 10^{-4} \mathrm{~s}$ in Section 3.3. Related to the fine mesh is also the requirement for often performing a re-mesh to maintain a high quality mesh.

\section{Conclusion}

Comparing the two ways of solving the bearing problem and two different boundary conditions yields:

- A clear influence from inlet type on pressure distribution for the NavierStokes model is seen. By varying the groove height as 1, 5 and 20 times the radial clearance a study of the influence of the depth is carried out. The more simple boundary conditions are not capable of rendering the same influence for the Reynolds equation. With a reduced groove height the Navier-Stokes solution approaches the Reynolds solution.

- Similar journal orbits are found for the four combinations of discretisation and groove design, in pairs based on the groove design. The shape of the orbit is maintained, but larger eccentricities are seen with increased groove height. For the Navier-Stokes solution the difference is as much as 0.2 times the radial clearance for groove heights of 1 times and 20 times the radial clearance, respectively. The Reynolds solution is close to the medium height of 5 times the radial clearance.

- A friction loss prediction being based on journal orbit is showing good agreement for the Reynolds and Navier-Stokes solutions. The friction loss is proportional to the groove height and also directly related to the journal orbit through the film thickness.

- Various flow phenomena occur depending on groove height.

\section{Acknowledgements}

The work is partly financed through the EU project HERCULES-C, grant agreement no. SCP1-GA-2011-284354. 


\section{References}

[1] O. Reynolds, On the theory of lubrication and its application to Mr. Beauchamp Tower's experiments, including an experimental determination of the viscosity of olive oil, Philosophical Transactions of the Royal Society of London 177 (1886) 157-234, DOI: 10.1098/rspl.1886.0021.

[2] A. Sommerfeld, Zur hydrodynamischen theorie der schmiermittelreibung, Zeit. angew. Math. u. Physik 50 (1904) 97-155, DOI: 10.1007/BF01657589.

[3] F. W. Ocvirk, Short-bearing approximation for full journal bearings, Technical note 2808, Cornell University (1952).

[4] H. W. Swift, The stability of lubricating films in journal bearings, in: Proceedings Institute Civil Engineers, Vol. 233, Thomas Telford, 1932, pp. 267-288.

[5] W. Stieber, Das Schwimmlager: Hydrodynamische Theorie des Gleitlagers, VDI-Verlag, GmbH., 1933.

[6] H. Elrod, M. Adams, A computer program for cavitation and starvation problems, Cavitation and related phenomena in lubrication (1974) 3741.

[7] D. Brewe, Theoretical modeling of the vapor cavitation in dynamically loaded journal bearings, Journal of Tribology 108 (4) (1986) 628-637, DOI: $10.1115 / 1.3261288$.

[8] P. Tucker, P. Keogh, A generalized computational fluid dynamics approach for journal bearing performance prediction, Proceedings of the Institution of Mechanical Engineers, Part J: Journal of Engineering Tribology 209 (2) (1995) 99-108, DOI: 10.1243/PIME_PROC_1995_209_412_02.

[9] K. Gertzos, P. Nikolakopoulos, C. Papadopoulos, CFD analysis of journal bearing hydrodynamic lubrication by Bingham lubricant, Tribology International 41 (12) (2008) 1190-1204, DOI: 10.1016/j.triboint.2008.03.002. 
[10] M. Schmidt, P. Stücke, M. Nobis, Numerical meshing issues for threedimensional flow simulations in journal bearings, in: 3rd Micro and Nano Flows Conference, 2011, Thessaloniki, Greece, 22-24 August 2011.

[11] M. Riedel, M. Schmidt, P. Stücke, Numerical investigation of cavitation flow in journal bearing geometry, in: EPJ Web of Conferences 45, 01081 (2013), 2013, DOI: 10.1051/epjconf/20134501081.

[12] M. Riedel, M. Schmidt, P. Reinke, M. Nobis, M. Redlich, Application of computational fluid dynamics on cavitations in journal bearings, in: EPJ Web of Conferences 67, 02099 (2014), 2014, DOI: 10.1051/epjconf/20146702099.

[13] F. Cheng, W. Ji, A velocity-slip model for analysis of the fluid film in the cavitation region of a journal bearing, Tribology International 97 (2016) 163-172, DOI: 10.1016/j.triboint.2016.01.025.

[14] Y. Song, C.-w. Gu, Development and validation of a three-dimensional computational fluid dynamics analysis for journal bearings considering cavitation and conjugate heat transfer, Journal of Engineering for Gas Turbines and Power 137 (12) (2015) 122502, DOI: 10.1115/1.4030633.

[15] X. Zhang, Z. Yin, G. Gao, Z. Li, Determination of stiffness coefficients of hydrodynamic water-lubricated plain journal bearings, Tribology International 85 (2015) 37-47, DOI: 10.1016/j.triboint.2014.12.019.

[16] M. Besanjideh, S. G. Nassab, Effect of lubricant compressibility on hydrodynamic behavior of finite length journal bearings running under heavy load conditions, Journal of Mechanics 32 (01) (2016) 101-111, DOI: $10.1017 /$ jmech.2015.51.

[17] CD-Adapco, User Guide STAR-CCM+ Version 10.02 (2015).

[18] B. J. Hamrock, S. R. Schmid, B. O. Jacobson, Fundamentals of fluid film lubrication, CRC press, 2004.

[19] M. Braun, W. Hannon, Cavitation formation and modelling for fluid film bearings: a review, Proceedings of the Institution of Mechanical Engineers, Part J: Journal of Engineering Tribology 224 (9) (2010) 839863, DOI: 10.1243/13506501JET772. 
[20] J. W. Lund, K. K. Thomsen, A calculation method and data for the dynamic coefficients of oil-lubricated journal bearings, in: Topics in Fluid Film Bearing and Rotor Bearing System Design and Optimization, ASME publ., 1978, pp. 1-28.

[21] C. K. Christiansen, P. Klit, J. H. Walther, A. Vølund, Orbit prediction of a journal bearing - finite element modelling and experimental studies, Finnish Journal of Tribology 33 (2015) 28-35, ISSN: 0780-2285. 


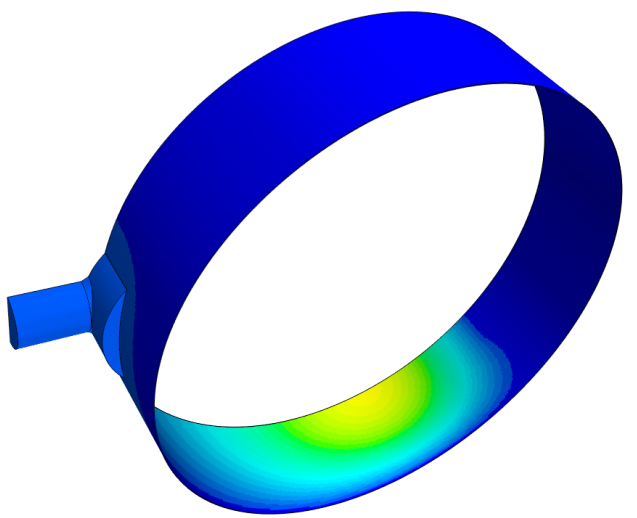

(a)

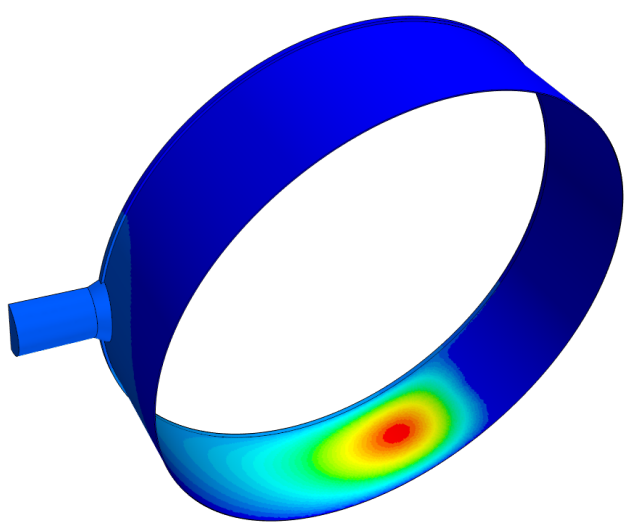

(c)

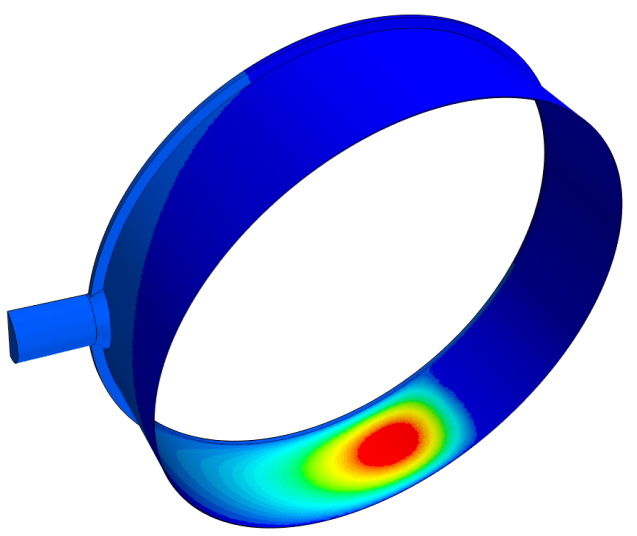

(b)

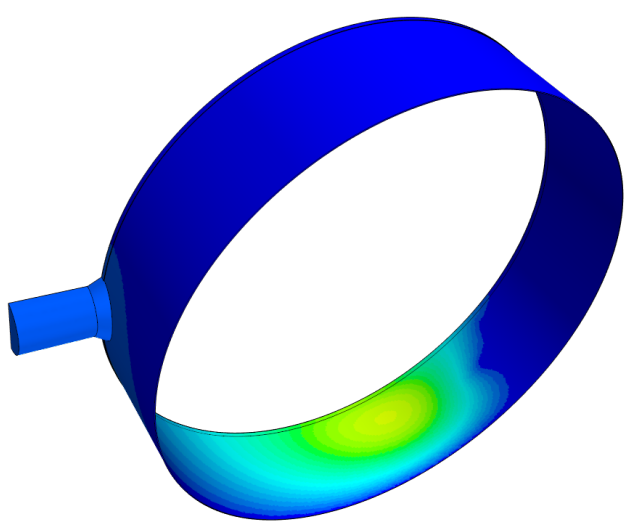

(d)

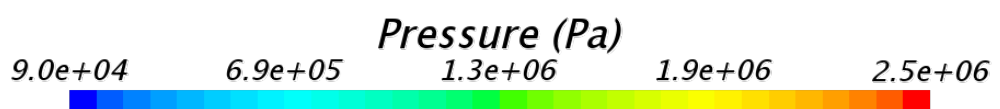

Figure 6: Pressure distribution of the four different groove designs; (a) is axial groove; (b) is circ. groove, $h=2 \mathrm{~mm}(20 \times C)$; (c) is circ. groove, $h=0.5 \mathrm{~mm}(5 \times C)$; and (d) is circ. groove, $h=0.1 \mathrm{~mm}(1 \times C)$. Time $t=0.3 \mathrm{~s}$ (maximum value of imposed load). 


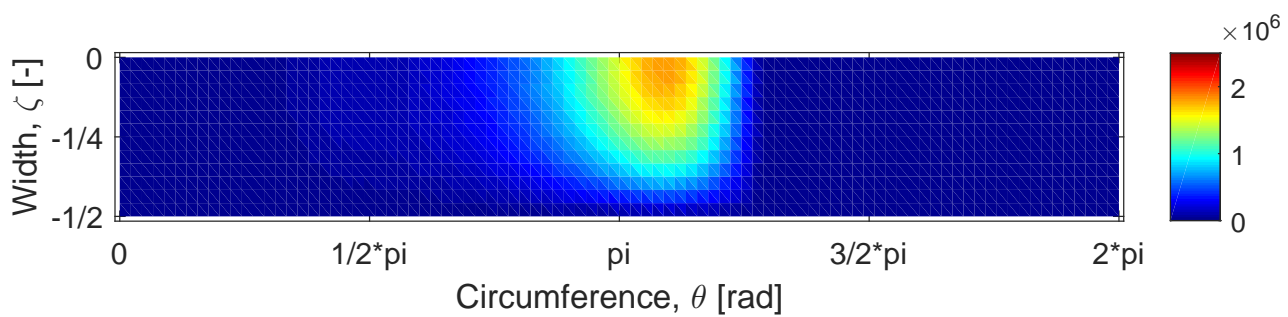

(a)

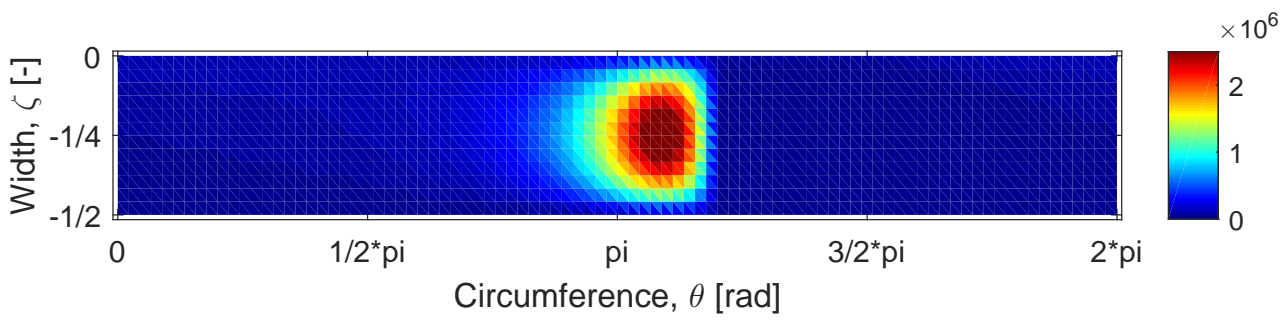

(b)

Figure 7: Pressure distributions using Reynolds equation and a mesh of $\theta \times \zeta$ of $(90 \times 24)$, (a) is axial groove, and (b) is circumferential groove. Time $t=0.3 \mathrm{~s}$ (maximum value of imposed load). 


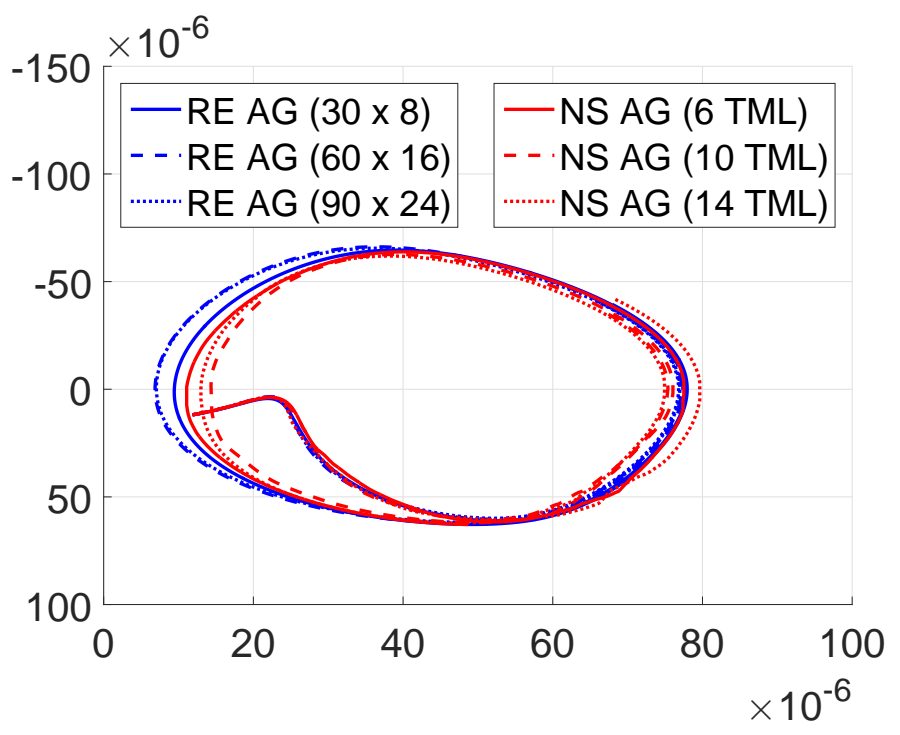

(a)

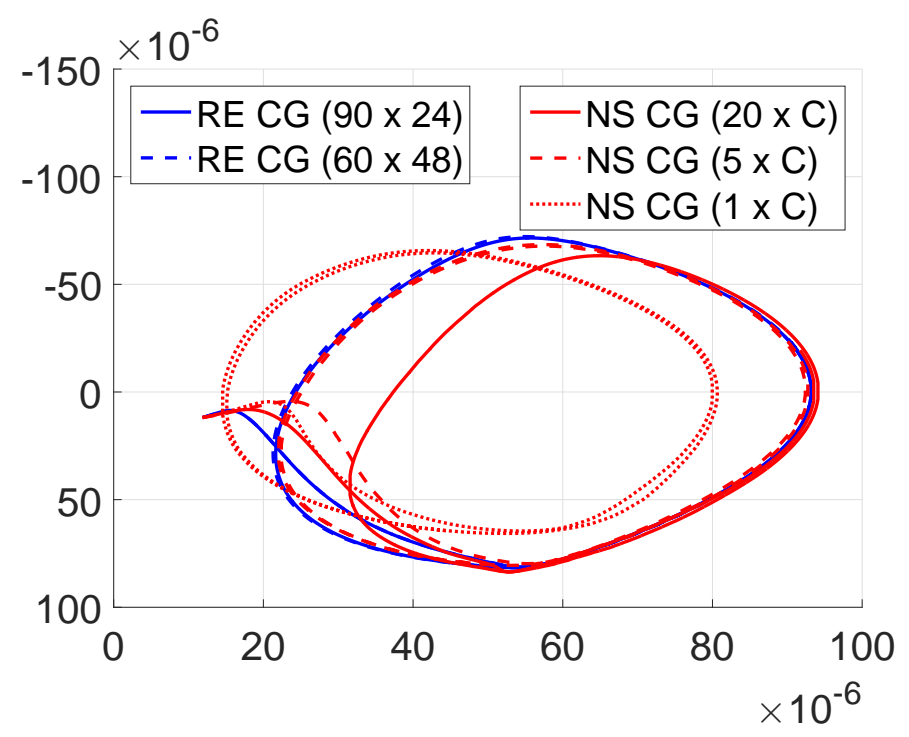

(b)

Figure 8: Journal orbits, (a) is axial groove (AG), and (b) is circumferential groove (CG). For the Reynolds equation (RE) the number in parenthesis designates the finite element mesh size (circ. times axial direction). For the Navier-Stokes (NS), TML is the number of thin mesher layers used in radial direction, and in case of the circ. groove the parenthesis indicates the groove height. Recall, that $C=118.75 \mu \mathrm{m}$ and cf. Figure 1a for the eccentricity and its components $\left(e_{x}, e_{y}\right)$. 


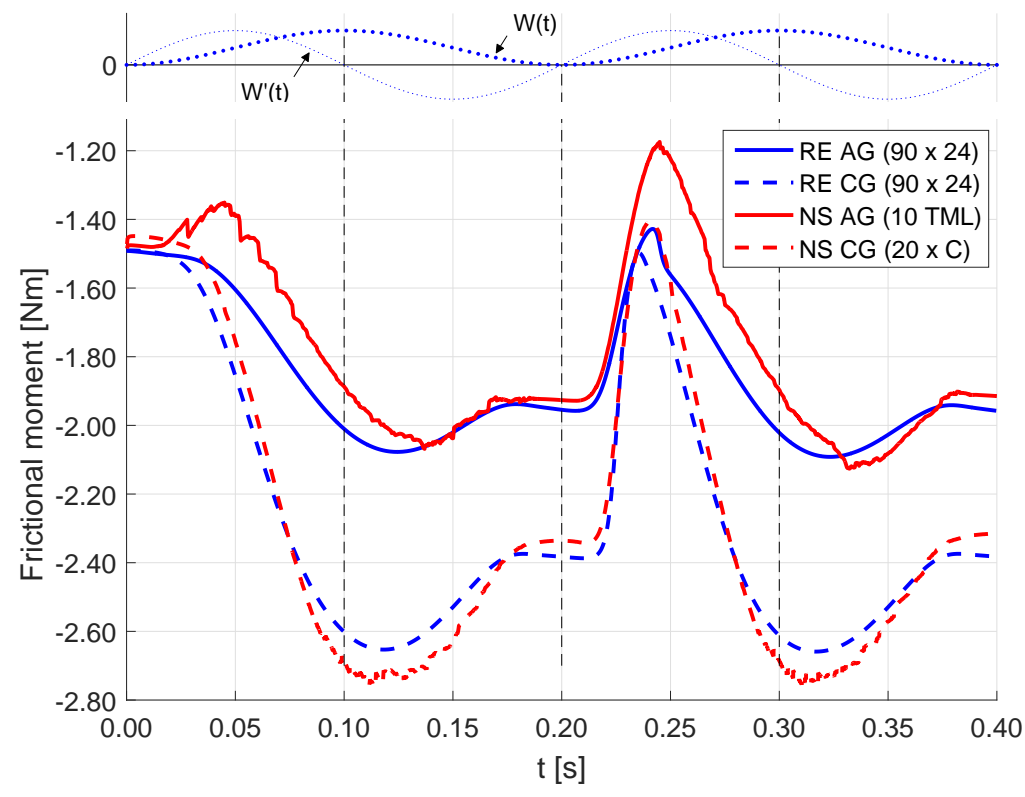

Figure 9: Frictional moment for the Reynolds and Navier-Stokes simulations. The course of the load curve and its time derivative is plotted above, for comparison of deviations. 


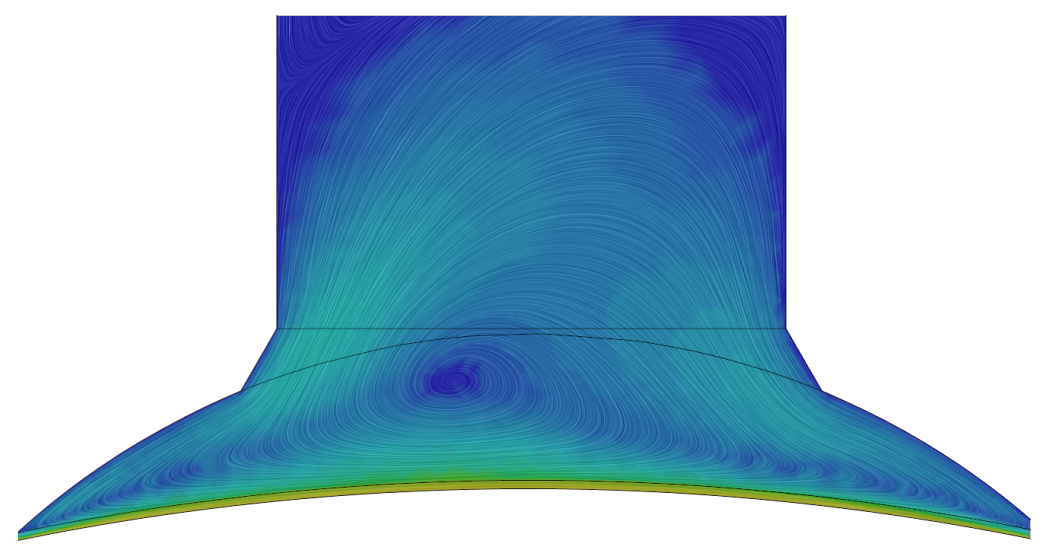

(a)

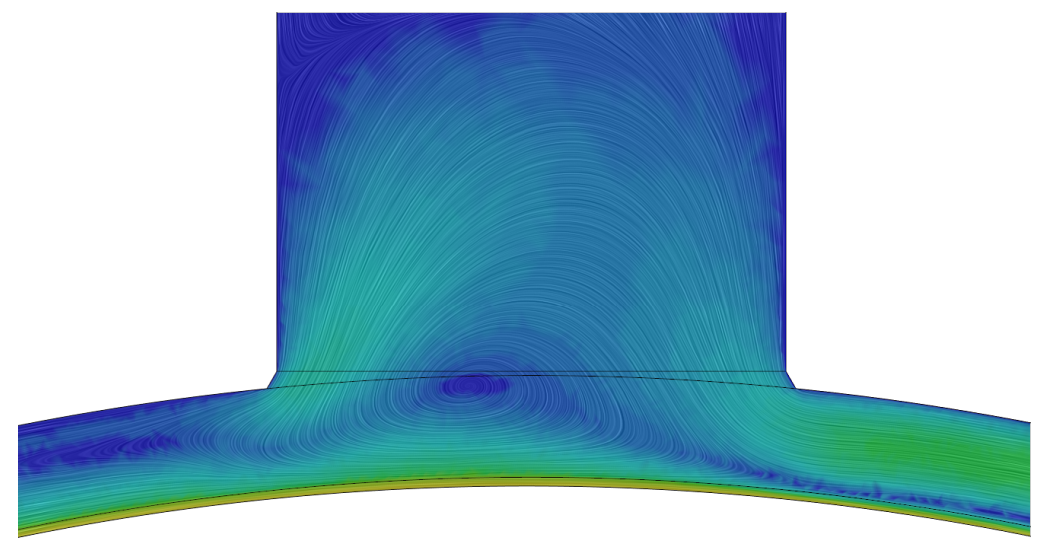

(b)

Figure 10: Velocity field in the symmetry plane at $t=0.3 \mathrm{~s}$ for the two bearing geometries: (a) Axial groove. (b) Circumferential groove with groove height $20 \times C$. Main journal rotation is CCW. Colour represents velocity magnitude with blue $=0 \mathrm{~m} / \mathrm{s}$ and khaki $=$ $3.14 \mathrm{~m} / \mathrm{s}$. 


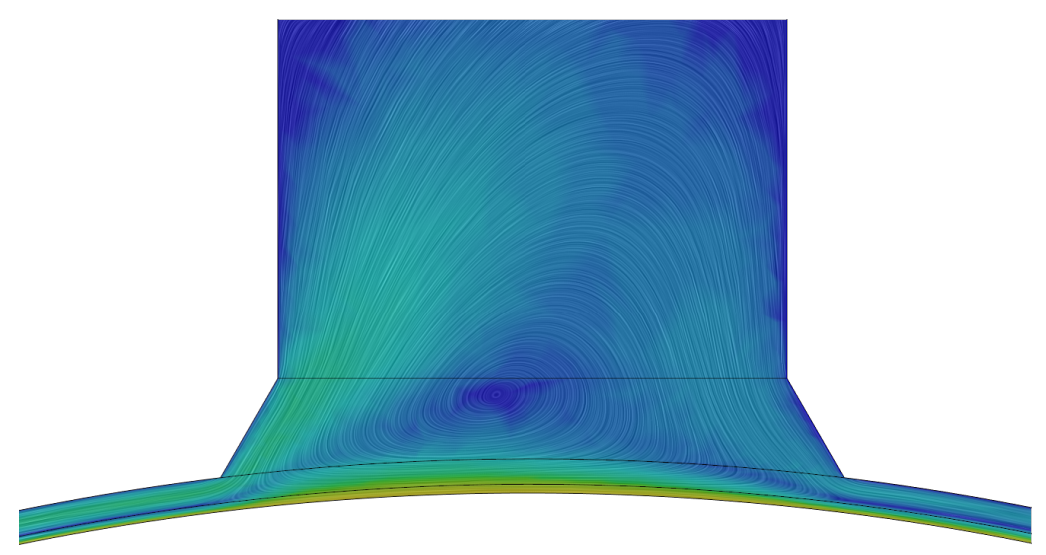

(c)

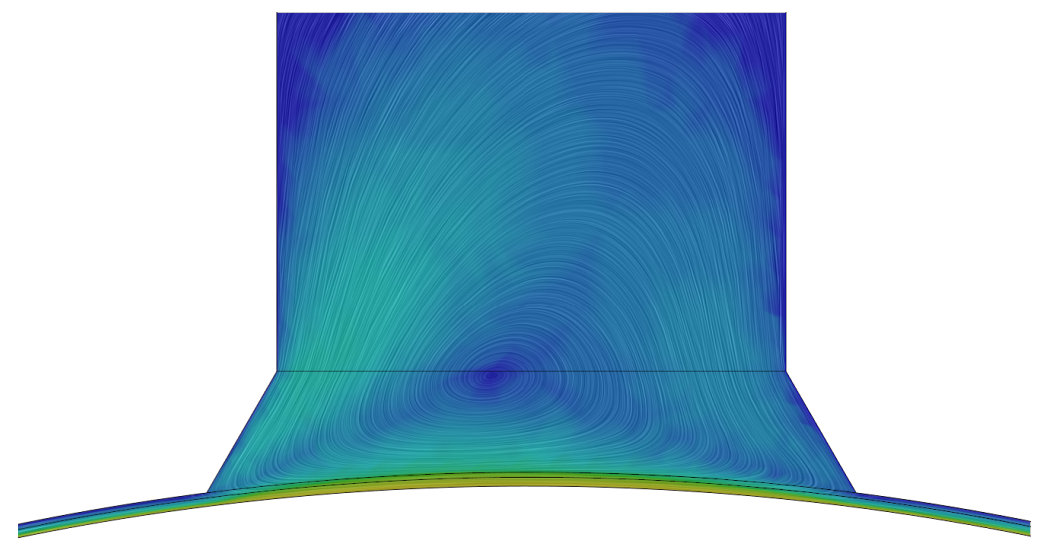

(d)

Figure 10: Velocity field in the symmetry plane at $t=0.3 \mathrm{~s}$ for the two bearing geometries: (c) Circumferential groove with groove height $5 \times C$. (d) Circumferential groove with groove height $1 \times C$. Main journal rotation is $\mathrm{CCW}$. Colour represents velocity magnitude with blue $=0 \mathrm{~m} / \mathrm{s}$ and $\mathrm{khaki}=3.14 \mathrm{~m} / \mathrm{s}$. 